\title{
Growth of Si ultrathin films on silver surfaces: Evidence of an $\mathrm{Ag}(110)$ reconstruction induced by $\mathrm{Si}$
}

\author{
Romain Bernard, ${ }^{1}$ Thomas Leoni, ${ }^{2}$ Axel Wilson, ${ }^{1}$ Tony Lelaidier, ${ }^{2}$ Houda Sahaf, ${ }^{2}$ Eric Moyen, ${ }^{2}$ Loïc Assaud, \\ Lionel Santinacci, ${ }^{2}$ Frédéric Leroy, ${ }^{2}$ Fabien Cheynis, ${ }^{2}$ Alain Ranguis, ${ }^{2}$ Haik Jamgotchian, ${ }^{2}$ Conrad Becker, ${ }^{2}$ \\ Yves Borensztein, ${ }^{1}$ Margrit Hanbücken, ${ }^{2}$ Geoffroy Prévot, ${ }^{1, *}$ and Laurence Masson ${ }^{2, \dagger}$ \\ ${ }^{1}$ CNRS, UMR 7588, Institut des Nanosciences de Paris, F-75005 Paris, France and UPMC Université Paris 06, UMR 7588, \\ Institut des Nanosciences de Paris, F-75005 Paris, France \\ ${ }^{2}$ Aix-Marseille Université, CNRS, CINaM UMR 7325, 13288 Marseille, France
}

(Received 18 July 2013; published 26 September 2013)

\begin{abstract}
We report here in situ measurements of the evolution of the $\mathrm{Ag}(110)$ surface during Si growth, using scanning tunneling microscopy and grazing incidence x-ray diffraction. We provide compelling evidence of an $\operatorname{Ag}(110)$ surface reconstruction associated with the release of $\mathrm{Ag}$ atoms induced by the growth of Si nanoribbons. Our results are in agreement with a missing row reconstruction of the Ag layer underneath the nanoribbons. This challenges the current understanding of the Si growth on nonreconstructed $\operatorname{Ag}(110)$, interpreted within the framework of silicene models.
\end{abstract}

DOI: 10.1103/PhysRevB.88.121411

PACS number(s): 81.07.-b, 61.05.cp, 68.37.Ef, 68.47.De

In the past five years, the $\mathrm{Si} / \mathrm{Ag}$ interface has proven to be an interesting system with potential intriguing properties. In a pioneering work, Leandri et al. ${ }^{1}$ have reported that upon submonolayer $\mathrm{Si}$ deposition at room temperature (RT) on the anisotropic $\operatorname{Ag}(110)$ surface, parallel, flat lying isolated nanoribbons (NRs) develop spontaneously. Sahaf et al. ${ }^{2}$ showed later that upon condensation at a substrate temperature ( $T_{\text {sub }}$ ) of $\sim 200^{\circ} \mathrm{C}$, these NRs form a one-dimensional (1D) array of parallel self-assembled nanoribbons (SANRs), with a pitch of $\sim 2 \mathrm{~nm}$. At completion, this $1 \mathrm{D}$ grating uniformly covers the entire substrate with a remarkably high degree of structural order.

These pioneering works have given rise to a number of theoretical and experimental studies concerning the formation and properties of ultrathin $\mathrm{Si}$ films on $\mathrm{Ag}$ substrates. A graphene-like signature in photoemission spectra has been reported on such films grown on $\mathrm{Ag}(110)$ and $\mathrm{Ag}(111)$ (Refs. 3 and 4) attributed to the formation of silicene, i.e., $s p^{2}$ bonded silicon atoms arranged in a two-dimensional (2D) honeycomb lattice. The SANR grating formed on $\mathrm{Ag}(110)$ has also been used as a novel Si template for the growth of identical highly ordered 1D nanostructures. $^{5-7}$

Concerning the atomic structure of the NRs, different models have been proposed, some of them being in favor of a Si honeycomb structure. ${ }^{8-12}$ All these models were based on the assumption that the 1D nanostructures are thin pure Si NRs, or nanostripes, on a nonreconstructed Ag substrate. This seemed reasonable considering that silver and silicon are nonmiscible materials as shown by the bulk phase diagram.

In this Rapid Communication, we demonstrate that none of these models is in agreement with the results presented here since the assumption of a nonreconstructed silver substrate has to be revised. By following in situ the Si growth on $\mathrm{Ag}(110)$, using scanning tunneling microscopy (STM) and grazing incidence X-ray diffraction (GIXD) for, respectively, Si deposition at RT and $190^{\circ} \mathrm{C}$, we provide compelling evidence that NR growth induces a surface reconstruction associated with the release of $\mathrm{Ag}$ atoms. Since these observations invalidate the proposed structural models based on a nonreconstructed substrate, this calls into question the recent description of
NRs as silicene NRs and consequently the origin of the Dirac cones previously observed by angle-resolved photoelectron spectroscopy.

All experiments were performed in setups working under ultrahigh vacuum (base pressure $10^{-10}$ Torr). The STM measurements were carried out at the INSP and at the CINaM using STM Omicron Nanotechnology systems. GIXD experiments were carried out on the ID3 beamline of the European Synchrotron Radiation Facility (ESRF) storage ring. The $\operatorname{Ag}(110)$ sample was prepared by repeated cycles of $\mathrm{Ar}^{+}$sputtering and annealing at $500^{\circ} \mathrm{C}$. To avoid any drift due to thermal radiation from the $\mathrm{Si}$ source installed in front of the STM stage, Si was evaporated from a thermally heated crucible using a commercial Omicron Nanotechnology e-beam evaporator. STM snapshot images of the same area were obtained after successive $\mathrm{Si}$ evaporations. During $\mathrm{Si}$ deposition, the tip was retracted by $\sim 1 \mu \mathrm{m}$ in order to avoid shading effect of the sample area behind the tip from the incoming evaporator beam. ${ }^{13}$ Residual drift has been carefully corrected using a homemade procedure. In addition to these specific experiments, further STM observations were made at liquid nitrogen temperature $\left(T_{\mathrm{LN} 2}\right)$ on NRs synthesized at RT and $190^{\circ} \mathrm{C}$. GIXD experiments have been carried out with $17 \mathrm{keV} \times$ rays. The incidence angle has been kept fixed at $0.22^{\circ}$. To define the surface basis, we have used the orthogonal vectors, expressed in the cubic basis of Ag, $\vec{a}_{x}=a(0,0,1), \vec{a}_{y}=a\left(\frac{1}{2},-\frac{1}{2}, 0\right)$, and $\vec{a}_{z}=a\left(\frac{1}{2}, \frac{1}{2}, 0\right)$, where $a=0.409 \mathrm{~nm}$. The Si flux has been calibrated from in situ X-ray reflectivity measurements and ex situ Rutherford backscattering spectrometry at the van de Graaf accelerator of the INSP, performed on thick Si deposits. One monolayer (ML) corresponds to the $\operatorname{Ag}(110)$ surface atom density.

The STM image presented in Fig. 1(f) shows isolated NRs grown on $\mathrm{Ag}(110)$ upon submonolayer Si deposition at RT. As already reported, ${ }^{14,15}$ most of these NRs, denoted hereafter single NRs (SNRs), appear as composed of two rows of round protrusions. The SNRs, randomly distributed on silver terraces, are perfectly aligned along the [11̄0] direction $(y)$ of the $\mathrm{Ag}(110)$ substrate with $\mathrm{a} \times 2$ periodicity along their edges $(\sim 0.6 \mathrm{~nm})$. All SNRs, varying only in length, possess the 

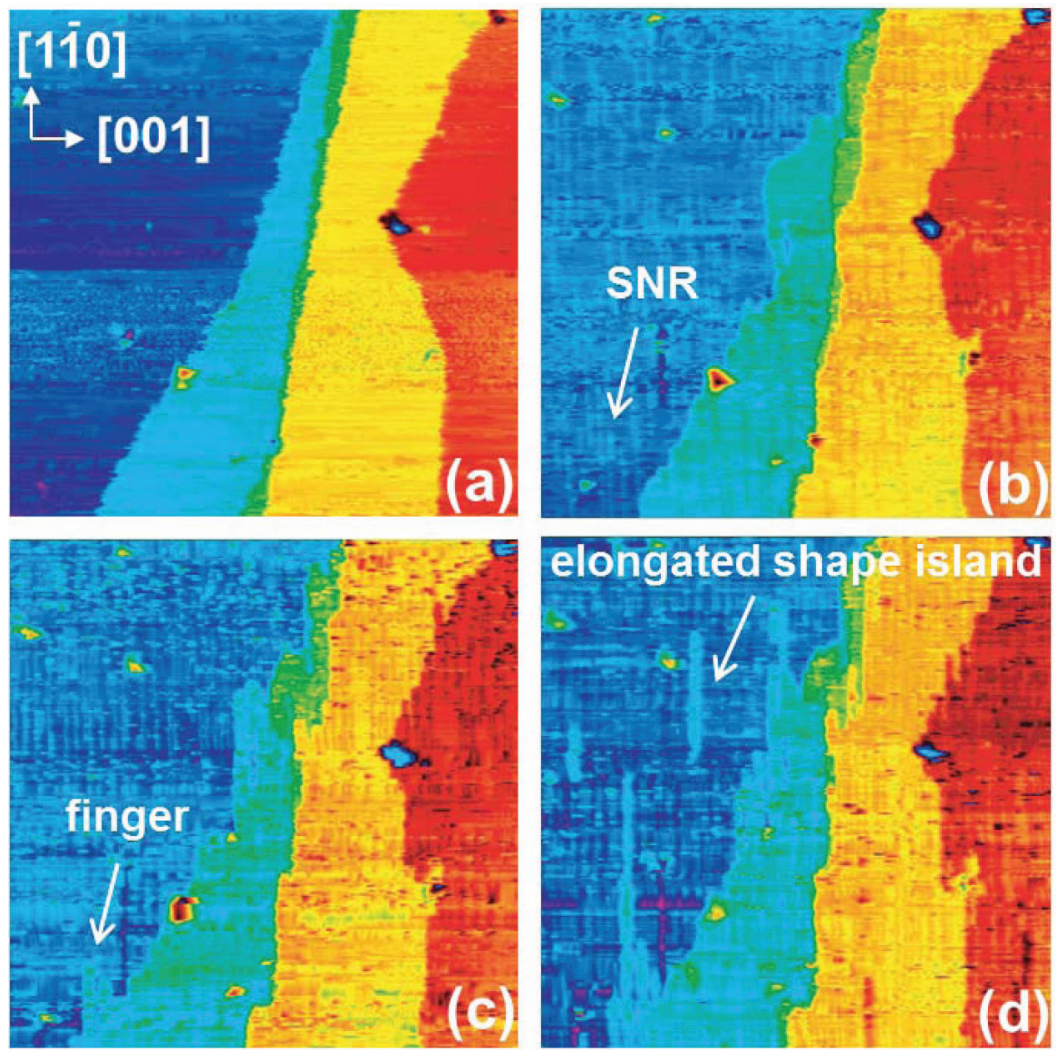

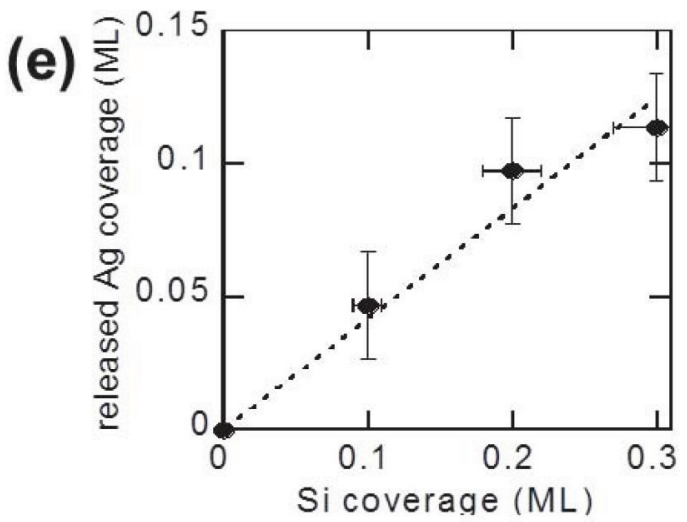

(f)

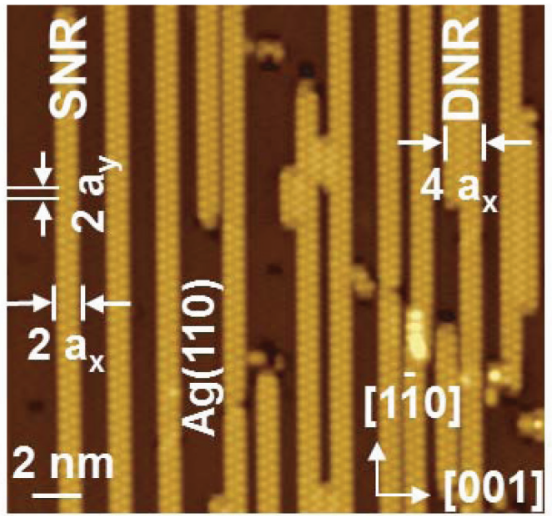

FIG. 1. (Color online) (a)-(d) Sequence of STM images $\left(234 \times 234 \mathrm{~nm}^{2}\right)$ of the same area showing the evolution of the Ag(110) surface upon Si deposition at RT. $I=510 \mathrm{pA}, V_{\text {sample }}=1.5 \mathrm{~V}$. (a) Bare silver surface. Colors correspond to the different terraces separated by monoatomic Ag steps. The upper terrace is at the right side of the image (red). (b) $\theta_{\mathrm{Si}}=0.1 \mathrm{ML}$. (c) $\theta_{\mathrm{Si}}=0.2 \mathrm{ML}$. (d) $\theta_{\mathrm{Si}}=0.3 \mathrm{ML}$. (e) evolution of the density of $\mathrm{Ag}$ atoms incorporated as a function of the Si coverage. (f) STM image recorded at $T_{\mathrm{LN} 2}$ of nanoribbons grown on $\operatorname{Ag}(110)$ upon Si deposition at RT. $\theta_{\mathrm{Si}} \sim 0.3 \mathrm{ML}$. I $=300 \mathrm{pA}, V_{\text {sample }}=1 \mathrm{~V}$.

same width of $2 a_{x}(\sim 0.8 \mathrm{~nm})$ and the same apparent height. Few of the observed NRs present a width of $4 a_{x}(\sim 1.6 \mathrm{~nm})$, corresponding to four rows of protrusions. We emphasize that these NRs differ only in width from the single ones and will be denoted hereafter double NRs (DNRs).

Figures 1(a)-1(d) show a series of four large scale STM images of the bare surface and the same surface area immediately after three successive $\mathrm{Si}$ depositions performed in the same conditions at RT. Figure 1(a) corresponds to the bare $\mathrm{Ag}(110)$ surface and shows flat terraces separated by monoatomic steps. Some portions of steps, close to the [1 10 ] direction, are straight while others, pinned by defects or impurities, are misoriented from this dense direction. Steps appear slightly frizzy due to adatoms moving along the step edges. After the first $\mathrm{Si}$ evaporation corresponding to $\theta_{\mathrm{Si}}=0.1 \mathrm{ML}$, a clear motion of the step edges is visible in Fig. 1(b), leading to an increase of their meandering. Isolated NRs, parallel to the straight parts of steps can also be distinguished. After the second $\mathrm{Si}$ deposition, the deformation of steps is more pronounced [see Fig. 1(c)]. Misoriented parts of step edges appear serrated with the formation of fingers. Upon further Si evaporation, these fingers continue to grow and additional elongated shape islands form on the terraces [see Fig. 1(d)].

The detailed view in Figs. 2(a) and 2(b) provides valuable information on the features observed. These STM images have been recorded after the second and the third Si depositions
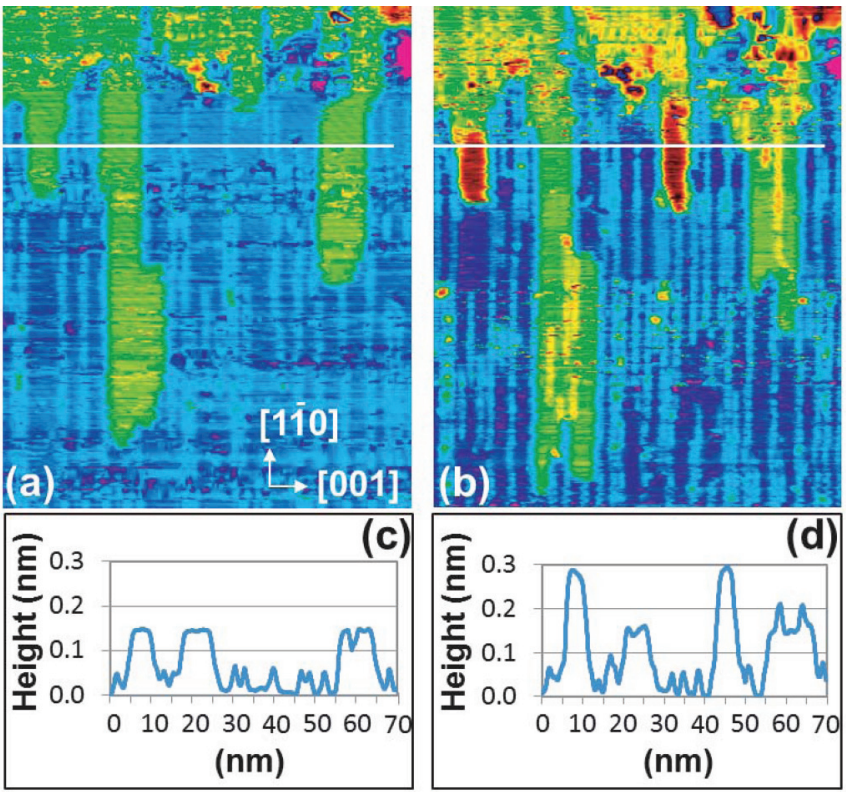

FIG. 2. (Color online) (a),(b) STM images $\left(75 \times 82 \mathrm{~nm}^{2}\right)$ showing the growth of fingers from steps perpendicular to the NRs upon Si deposition at RT. $I=510 \mathrm{pA}, V_{\text {sample }}=1.5 \mathrm{~V}$. (a) $\theta_{\mathrm{Si}}=0.2 \mathrm{ML}$. (b) $\theta_{\mathrm{Si}}=0.3$ ML. (c) and (d) Height profiles along the white transverse lines in (a) and (b), respectively. 

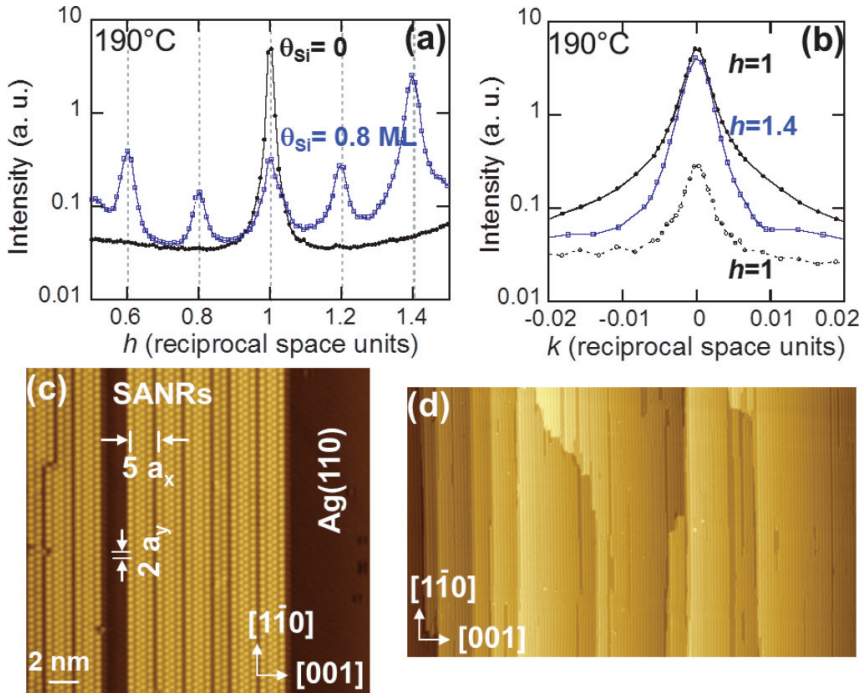

FIG. 3. (Color online) (a) $h$ scans for $k=0$ and $l=0.05$ recorded at $T_{\text {sub }}=190^{\circ} \mathrm{C}$ during $\mathrm{Si}$ deposition. Black dots: bare $\mathrm{Ag}$ surface. Blue squares: $\theta_{\mathrm{Si}}=0.8 \mathrm{ML}$. (b) $k$ scan for $l=0.05$ and $h=1$ (black dots) corresponding to the bare surface (filled dots) and $\theta_{\mathrm{Si}}=0.8$ ML (open dots), and for $h=1.4$ corresponding to $\theta_{\mathrm{Si}}=0.8 \mathrm{ML}$ (blue squares). (c) and (d) STM images recorded at $T_{\mathrm{LN} 2}$ after $\mathrm{Si}$ deposition at $190{ }^{\circ} \mathrm{C}$. (c) $\theta_{S i} \sim 0.5 \mathrm{ML} . I=500 \mathrm{pA}, V_{\text {sample }}=1 \mathrm{~V}$. (d) $\left(280 \times 170 \mathrm{~nm}^{2}\right) . \theta_{\mathrm{Si}} \sim 0.8 \mathrm{ML} . I=130 \mathrm{pA}, V_{\text {sample }}=0.63 \mathrm{~V}$.

[Figs. 1(c) and 1(d)] on another part of the probed area. They clearly show that fingers have grown between NRs. The height profile presented in Fig. 2(c) indicates that fingers in Fig. 2(a) have heights very close to one silver layer $(\sim 0.145 \mathrm{~nm})$. Moreover, in Fig. 2(d), these fingers appear to be covered either by a second silver layer (total height $\sim 0.29 \mathrm{~nm}$ ) or by SNRs. This demonstrates that these fingers correspond to $\mathrm{Ag}(110)$ terraces and result from incorporation of extra silver adatoms at step edges. In the same way, height profiles of elongated shape islands observed in Fig. 1(d) indicate that they are composed of Ag. Measuring the additional Ag matter in Figs. 1(a)-1(d) and using the calibration of the Si flux, we have measured that $(0.42 \pm 0.15) \mathrm{Ag}$ atoms are incorporated at step edges or new islands per Si incoming atom [see Fig. 1(e)]. These extra Ag atoms are released during Si growth. For a low NR density, they diffuse and are incorporated at step edges, inducing the motion of the steps. As the NR density increases, steps are partially blocked by NRs, leading to the formation of growing fingers. When a high density of NRs prevents them from reaching the steps, Ag atoms nucleate on the terraces and form elongated shape islands.

As $T_{\text {sub }}$ increases, the ratio between DNRs and SNRs increases. At $T_{\text {sub }}=190^{\circ} \mathrm{C}$, essentially DNRs form upon $\mathrm{Si}$ deposition. Moreover, they self-assemble in a $\times 5$ periodicity in the [001] direction, perpendicular to the so-called SANRs [see Fig. 3(c)]. Large scale STM observations show that the surface roughness does not significantly increase during $\mathrm{Si}$ growth [see Ref. 6 and Fig. 3(d)]. Up to completion of the SANR grating, the high mobility of Ag adatoms at $190^{\circ} \mathrm{C}$ and the low density of SANR domains prevent the formation of serrated steps or Ag islands. From the calibration of the $\mathrm{Si}$ flux, we find that completion is obtained for a Si coverage $\theta_{\mathrm{Si}}=\theta_{\mathrm{SANR}}=0.8 \pm 0.2 \mathrm{ML}$.
At $190^{\circ} \mathrm{C}$, we were not able to follow with STM the evolution of the morphology of the same surface area upon successive Si evaporations due to the fast motion of steps at the time scale of our experiments. However, complementary results could be obtained from GIXD measurements, taking advantage of the high structural order of the growing $\mathrm{Si}$ ultrathin film at $190^{\circ} \mathrm{C}$. At this deposition temperature, as soon as the evaporation begins, $\mathrm{Si}$ organizes into $2 \mathrm{D}$ islands consisting of SANRs locally packed in a $\times 5$ periodicity that give rise to diffraction rods at fractional positions $h=$ $p / 5 \pm 0.002$ ( $p$ integer) for integer values of $k$. Scans along the $h$ and $k$ directions are presented in Figs. 3(a) and 3(b) for the bare surface and at completion of the $\times 5$ grating. The diffraction peaks associated with the $5 a_{x}$ periodicity are very narrow, with a FWHM of 0.033 and 0.003 along the $h$ and $k$ directions, respectively [corresponding values of 0.005 and 0.003 are measured for the $(1,0,0.05)$ anti-Bragg peak for the bare surface]. The FWHM along $h$ corresponds to a mean domain size of $\sim 12 \mathrm{~nm}$ in agreement with STM observations [see Fig. 3(d)]. Along the [110] direction, domains spread on the whole terraces.

Figure 4(a) shows the evolution, during $\mathrm{Si}$ deposition at $190^{\circ} \mathrm{C}$, of the intensity at $(1.4,0,0.05)$ and $(1,0,0.05)$, corresponding, respectively, to a superstructure and a substrate anti-Bragg reflection. They have been computed by integration along the $h$ and $k$ directions of the scans performed during $\mathrm{Si}$ deposition and have been corrected from geometrical factors. ${ }^{16}$ The increase of the intensity of the reflection associated with the growth of the SANRs is clearly observed until completion is reached. The most striking feature is the variation of the intensity at the $(1,0,0.05)$ position. As shown in Fig. 4(a), its value strongly decreases as the SANR grating grows until it almost vanishes at completion of the ultrathin film. Upon further Si deposition, we observe a reincrease of this value, correlated to the decrease of the intensity associated with the diffraction by the $\times 5$ grating. Since the intensities are obtained by integration in the $(h, k)$ plane, this decay cannot be attributed to any large scale roughness or domain size effects, and therefore must be associated with the atomic structure of SANRs that progressively cover the whole surface, including possible structural modification of the Ag surface underneath.

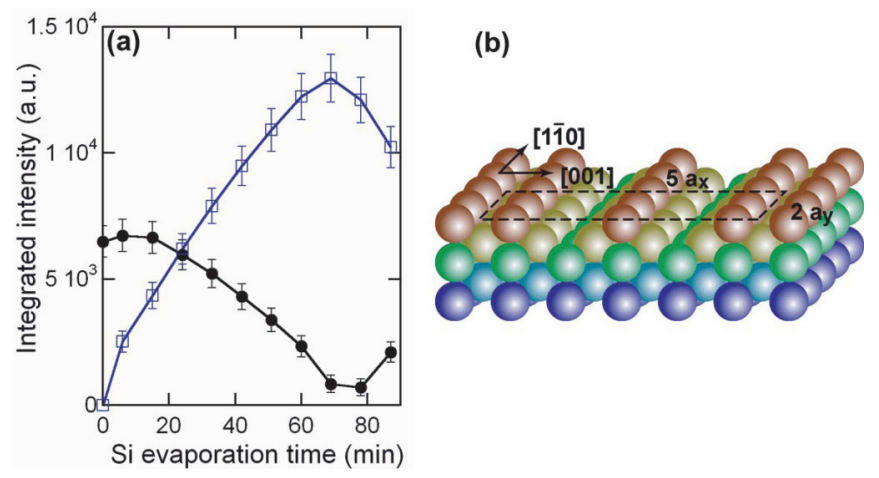

FIG. 4. (Color online) (a) Evolution, during Si deposition at $T_{\text {sub }}=190^{\circ} \mathrm{C}$, of the integrated intensities associated with the $\operatorname{Ag}(110)$ surface at $(1,0,0.05)$ (black dots) and with the $\times 5$ superstructure at $(1.4,0,0.05)$ (blue squares). (b) Model of the $(5 \times 2)$ reconstruction of the $\mathrm{Ag}(110)$ surface induced by the SANR growth. 
These SANRs give rise to intense diffraction satellites. In particular, the intensity of the $(1.4,0,0.05)$ reflection is, at completion, twice the intensity measured on the bare surface at the anti-Bragg $(1,0,0.05)$ position.

For a given reflection, corresponding to a scattering wave vector $\vec{q}$, the intensity measured after correction of geometrical factors is the square of the amplitude of the structure factor $F(\vec{q}) . F(\vec{q})$ is given by the scattering of the substrate and layer atoms:

$$
\begin{aligned}
F(\vec{q}) & =A_{0}\left|A_{\mathrm{Ag}}(\vec{q})+A_{\mathrm{Si}}(\vec{q})\right| \\
& =A_{0}\left|\sum_{\mathrm{Ag}} f_{\mathrm{Ag}}(q) e^{i \vec{q} \cdot \vec{r}_{n}-\alpha z_{n}}+\sum_{\mathrm{Si}} f_{\mathrm{Si}}(q) e^{i \vec{q} \cdot \vec{r}_{n}}\right|,
\end{aligned}
$$

where $\vec{r}_{n}$ are the atomic positions, and $f_{\mathrm{Ag}}$ and $f_{\mathrm{Si}}$ are the atomic scattering factors for $\mathrm{Ag}$ and $\mathrm{Si}$ atoms. $\alpha$ is the attenuation length of the $\mathrm{x}$ rays in the crystal $(z>$ $0)$. At $17 \mathrm{keV}, f_{\mathrm{Ag}}(1,0,0.05)=42.7, f_{\mathrm{Si}}(1,0,0.05)=11.5$, $f_{\mathrm{Ag}}(1.4,0,0.05)=39.9$, and $f_{\mathrm{Si}}(1.4,0,0.05)=10.3{ }^{17}$ For a bare $\mathrm{Ag}(110)$ surface, neglecting Debye-Waller factors, it can be easily shown that the summation of all crystal atoms leads to $F(1,0,0) \approx A_{0} N_{x} N_{y}\left(f_{\mathrm{Ag}} / 2\right)$, where $N_{x}$ and $N_{y}$ are the number of $(1 \times 1)$ unit cells in the directions parallel to the surface within the coherence length of the x-ray beam. We define $\tilde{F}$ as $F / A_{0} N_{x} N_{y}$. As shown by Eq. (1), for $\theta_{\text {SANR }}=0.8 \mathrm{ML}$, the contribution of Si atoms to $\tilde{F}$ cannot exceed, within the error bars $\pm 0.2 \mathrm{ML}$ associated with the coverage measurements, $(0.8+0.2) f_{\mathrm{Si}}=11.5$ for $(1,0,0.05)$ and 10.3 for $(1.4,0,0.05)$. It clearly demonstrates that $\mathrm{Si}$ atoms in the $\times 5$ superstructure cannot be responsible for either the observed decay by a factor of 3 of the $(1,0,0.05)$ structure factor nor for the increase of the $(1.4,0,0.05)$ structure factor. On the contrary, such behavior can result from the removal of $\mathrm{Ag}$ atoms in the surface plane.

The decay of the substrate anti-Bragg peak has been observed, for example, for $(3 \times 1)$ or $(5 \times 1)$ missing row reconstructions on $\operatorname{Pt}(110)$ (Refs. 18 and 19). It can be derived from Eq. (1) that for a bare $\mathrm{Ag}$ surface, neglecting all relaxations $\tilde{F}(1,0,0)=f_{\mathrm{Ag}}\left(1 / 2-\theta_{\mathrm{Ag}}\right)$, where $\theta_{\mathrm{Ag}}$ is the coverage of missing Ag atoms in the surface plane. Using the ROD program ${ }^{20}$ to calculate the $(h, 0,0.05)$ structure factors for various densities of the first Ag plane, random positions of $\mathrm{Si}$ atoms, and taking into account possible substrate relaxations, the best agreement is obtained for two missing $\mathrm{Ag}$ rows within a $(5 \times 2)$ unit cell [see Ref. 21 and Fig. 4(b)]. From the experimental data set, it has not, however, been possible to assign unambiguously atomic positions for $\mathrm{Si}$ atoms, due to their low atomic scattering factor as compared to Ag atoms.

Such Ag reconstruction is at first sight surprising since $\mathrm{Ag}$ and $\mathrm{Si}$ are known to form an abrupt interface and bare $\mathrm{Ag}(110)$ does not show any reconstruction under UHV. Actually, $4 d$-band $\operatorname{Ag}(110)$ is known to be an intermediate case of noble metals between the unreconstructed $3 d$-band $\mathrm{Cu}(110)$ and the missing row reconstructed $5 d$-band $\mathrm{Au}(110){ }^{22} \mathrm{Sev}$ eral theoretical and experimental studies have reported the possibility to induce $(n \times 1)$ missing row reconstructions of this surface upon weak elastic deformations ${ }^{23}$ or alkali-metal adsorption. ${ }^{24}$ The model displayed in Fig. 4(b) corresponds to one Ag atom released for two Si atoms in the SANRs. This is, within the experimental uncertainties, in good agreement with RT measurements. We can thus expect that a missing row reconstruction also forms at RT, each SNR being associated with the removal of one Ag row in the surface plane. However, we cannot exclude more complex atomic structures associated with a lower Ag density in the (110) surface plane.

In summary, our STM and GIXD measurements evidence a structural modification of the $\mathrm{Ag}(110)$ substrate induced by the growth of NRs upon Si deposition at RT and $190^{\circ} \mathrm{C}$. Our STM and GIXD results demonstrate the removal of part of the $\mathrm{Ag}$ atoms of the $\mathrm{Ag}(110)$ surface and are in agreement with a missing row reconstruction of the Ag layer underneath the NRs. We assert that our results provide key elements for the determination of the exact atomic structure of the first system which has been interpreted within the framework of silicene models.

The authors thank A. Saúl for fruitful discussions. Support from the ID3 beamline staff at ESRF is greatly acknowledged.
*Corresponding author: prevot@insp.jussieu.fr

${ }^{\dagger}$ Corresponding author: masson@ cinam.univ-mrs.fr

${ }^{1}$ C. Leandri, G. Le Lay, B. Aufray, C. Girardeaux, J. Avila, M. E. Dávila, M. C. Asensio, C. Ottaviani, and A. Cricenti, Surf. Sci. 574, L9 (2005).

${ }^{2}$ H. Sahaf, L. Masson, C. Léandri, B. Aufray, G. Le Lay, and F. Ronci, Appl. Phys. Lett. 90, 263110 (2007).

${ }^{3}$ P. De Padova, C. Quaresima, C. Ottaviani, P. M. Sheverdyaeva, P. Moras, C. Carbone, D. Topwal, B. Olivieri, A. Kara, H. Oughaddou, B. Aufray, and G. Le Lay, Appl. Phys. Lett. 96, 261905 (2010).

${ }^{4}$ P. Vogt, P. De Padova, C. Quaresima, J. Avila, E. Frantzeskakis, M. C. Asensio, A. Resta, B. Ealet, and G. Le Lay, Phys. Rev. Lett. 108, 155501 (2012).

${ }^{5}$ F. Dettoni, H. Sahaf, E. Moyen, L. Masson, and M. Hanbücken, Europhys. Lett. 94, 28007 (2011).

${ }^{6}$ L. Masson, H. Sahaf, P. Amsalem, F. Dettoni, E. Moyen, N. Koch, and M. Hanbücken, Appl. Surf. Sci. 267, 192 (2013).
${ }^{7}$ P. De Padova, C. Ottaviani, F. Ronci, S. Colonna, B. Olivieri, C. Quaresima, A. Cricenti, M. E. Dávila, F. Hennies, A. Pietzsch, N. Shariati, and G. Le Lay, J. Phys.: Condens. Matter 25, 14009 (2013).

${ }^{8}$ Guo-min He, Phys. Rev. B 73, 035311 (2006).

${ }^{9}$ H. Sahaf, C. Léandri, E. Moyen, M. Macé, L. Masson, and M. Hanbücken, Europhys. Lett. 86, 28006 (2009).

${ }^{10}$ B. Aufray, A. Kara, S. Vizzini, H. Oughaddou, C. Léandri, B. Ealet, and G. Le Lay, Appl. Phys. Lett. 96, 183102 (2010).

${ }^{11}$ A. Kara, S. Vizzini, C. Léandri, B. Ealet, H. Oughaddou, B. Aufray, and G. Le Lay, J. Phys.: Condens. Matter 22, 45004 (2010).

${ }^{12}$ C. Lian and J. Ni, Physica B 407, 4695 (2012).

${ }^{13}$ B. Voigtländer, Surf. Sci. Rep. 43, 127 (2001).

${ }^{14}$ F. Ronci, S. Colonna, A. Cricenti, P. De Padova, C. Ottaviani, C. Quaresima, B. Aufray, and G. Le Lay, Phys. Status Solidi C 7, 2716 (2010).

${ }^{15}$ S. Colonna, G. Serrano, P. Gori, A. Cricenti, and F. Ronci, J. Phys.: Condens. Matter 25, 315301 (2013). 
${ }^{16}$ O. Robach, Y. Garreau, K. Aïd, and M. B. Véron-Jolliot, J. Appl. Crystallogr. 33, 1006 (2000).

${ }^{17}$ P. J. Brown, A. G. Fox, E. N. Maslen, M. A. O'Keefe, and B. T. M. Willis, International Tables for Crystallography 100, 554 (2006).

${ }^{18}$ I. K. Robinson, P. J. Eng, C. Romainczyk, and K. Kern, Phys. Rev. B 47, 10700 (1993).

${ }^{19}$ I. K. Robinson, M. C. Saint-Lager, P. Dolle, S. Boutet, M. De Santis, and R. Baudoing-Savois, Surf. Sci. 575, 321 (2005).

${ }^{20}$ E. Vlieg, J. Appl. Crystallogr. 33, 401 (2000).

${ }^{21}$ See Supplemental Material at http://link.aps.org/supplemental/ 10.1103/PhysRevB.88.121411 for comparison between experimen- tal and theoretical structure factors and for details on the fitting procedure.

${ }^{22}$ S. Olivier, A. Saúl, and G. Tréglia, Appl. Surf. Sci. 212-213, 866 (2003).

${ }^{23}$ M. Guillopé and B. Legrand, Surf. Sci. 215, 577 (1989).

${ }^{24}$ B. E. Hayden, K. C. Prince, P. J. Davie, G. Paolucci, and A. M. Bradshaw, Solid State Commun. 48, 325 (1983); J. W. M. Frenken, R. L. Krans, J. F. van der Veen, E. Holub-Krappe, and K. Horn, Phys. Rev. Lett. 59, 2307 (1987); F. Moresco, M. Rocca, T. Hildebrandt, V. Zielasek, and M. Henzler, Surf. Sci. 424, 62 (1999). 